\title{
Analysis of interface usability evaluation using digital measurement tools*
}

\author{
Xinyun Pan $^{1 \mathrm{a} \dagger}$, Edwin Kofi Nyefrer Donkor ${ }^{1 \mathrm{~b}}$, Chunfa Sha ${ }^{1 \mathrm{\dagger}}$ \\ ${ }^{1}$ College of Arts, Jiangsu University, Zhenjiang, Jiangsu, China
}

\begin{abstract}
Based on the articles on interface usability collected by CNKI and Web of Science (WOS), the current research status, trends, and recommendations on interface usability were analyzed in terms of the number of articles published, literature sources, institutions, authors, and keywords by combining the CNKI and WOS bibliometric analysis tools and CiteSpace information visualization software. Using digital technology methods such as bibliometric analysis, data visualisation and knowledge mapping analysis, the following conclusions were drawn. Research on interface usability mainly focuses on usability testing and interface optimization. At present, the research methods of interface usability are relatively mature, and the evaluation direction of interface usability tends to combine the eye movement experiments, the subjective and objective evaluation of users. The use of digital tools to study the current situation, hotspots and trends in interface usability can provide advice and assistance in the design of relevant interfaces.
\end{abstract}

\section{Data sources and research methodology}

This paper comprehensively reviews the progress of usability research in recent decades, including the conceptual evolution, evaluation indexes and evaluation methods. It focuses on the analysis of the hot issues in this field and the relevant achievements in usability evaluation research in recent years.

\subsection{Data sources}

The literature data in this paper were obtained from China Knowledge Infrastructure (CNKI) and Web of Science citation index database in the Web of Science core database. Enter the search formula SU=('system usability' + 'interface' + 'APP')*'evaluation method'*'index' OR $\mathrm{SU}=($ 'system usability' + 'usability test')*'interface' in the CNKI professional search, and set the time span from 1 January 2000 - 4 April 2020,a total of 564 papers were obtained, excluding conference papers. The Web of Science core database uses the search formula $\mathrm{TS}=($ "usability evaluation") AND $\mathrm{TS}=$ (interface) AND $\mathrm{TS}=$ (usability testing) OR TS= ("interface evaluation" OR $\mathrm{TS}=$ ("interface usability") was used to search for relevant documents, and the same time span was set as 2000-2020. Removal of irrelevant literature such as conference papers and newsletters yielded a total of 236 relevant papers. This data pair was imported into citespace and the bibliometric platform, and slices were set to 2 years for further analysis.

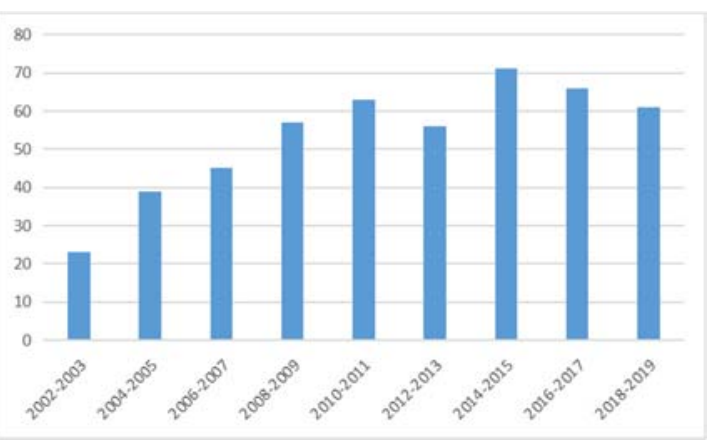

Figure 1: CNKI, Web of Science comprehensive year-on-year volume of literature

\subsection{Research methodology}

This paper combines a bibliometric analysis platform with CiteSpace to visualize and dissect the development of interface or system usability over the last 20 years, covering research methods such as bibliometric analysis, visualization, and knowledge graph analysis. CiteSpace is a tool that looks at the potential knowledge embedded in scientific analysis, and is a key component in the development of scientometrics, data visualization. It is a literature information visualization and analysis software gradually developed under the background of Drexel University. Developed by Drexel University Chinese scholar Chaomei Chen and his team, this software has a wide range of applications in identifying and detecting disciplinary frontiers, discovering research hotspots, etc. It is currently recognized as one of the most authoritative literature visualization and analysis software in international academic circles.

\footnotetext{
a919640313@qq.com

bgetteown@yahoo.com

c24679598@qq.com
} 


\subsection{Author, institutional partnerships}

In the analysis of the main publishing institutions in CiteSpace, there are three institutions with a high number of publications, namely, the School of Mechanics of Southeast University, the Institute of Psychology of the Chinese Academy of Sciences, and the Beijing Institute of Technology.

481 papers were imported into CiteSpace and subsequently authors and institutions were selected as Node Types for visualization and analysis. From the figure 3 , we can see that the research institutions in China are scattered, but there is a little amount of collaborations, which includes psychological research teams. The main researchers were made up of Jiang Ke, Pan Fei's team, Zhang Kan and Wu Changxu's team. Jiang Ke and Pan Fei mainly used eye movement experiment to evaluate and improve the relevant websites, and construct the interface usability evaluation model based on SUS scale. [1]. The team of Kan Zhang and Changxu Wu mainly researched the related methods of interface usability and the intrinsic cognitive influence of users, mostly in the form of review articles, which introduced usability methods [2]. Jiajie of the Department of Psychology summarized and reviewed the emotion test scale independently developed by the researchers to make usability testing more comprehensive and complete [3].
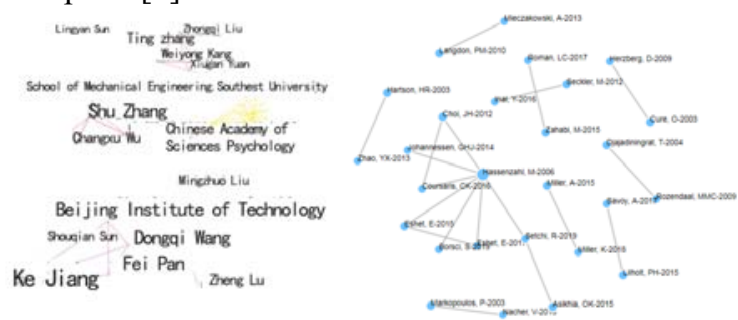

Figure 2: Institutional and national partnerships at home and abroad

Using bibliometric platform to analyze the collaborative relationship between the main research institutions and countries of foreign language literature travel imagery, we obtained Figure 4, which is based on the number of citations of the literature. In addition, the number of publications in Asian countries such as China and South Korea is also increasing rapidly. Generally speaking, the research field on interface usability is expanding, and the main research teams are concentrated in European countries.

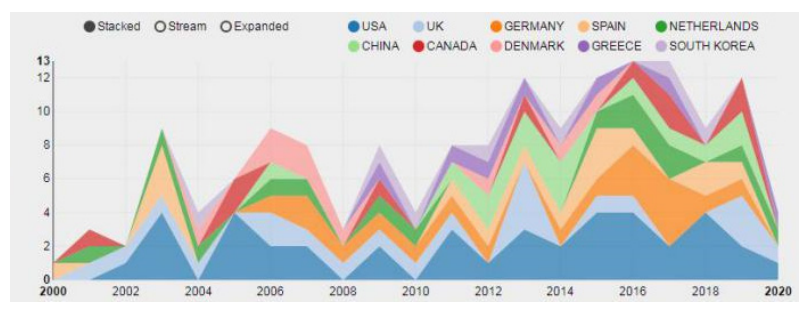

Figure 3: Changes in the number of articles by country over time

\section{Domestic and international research hotspots}

\subsection{Analysis of domestic research hotspots}

CiteSpace was used to analyze the keywords of major research papers on interface usability in China. According to the frequency of keywords, we can get the hotspots of interface usability research in China, as shown in Figure 5 and Table 2.

By the frequency of keywords and the core vocabulary clustering map, the high-frequency words were divided into two directions: the first category is user-oriented such as user-centered (UCD), user experience, subjective evaluation, mental models, etc., where user experience is an emergent keyword in 2018, lasting to 2020; the second category is usability testing, such as eye tracking and Hierarchical analysis method, indicator system, etc. The keywords that appeared after 2010 were more specific than before. For Example, the design term of the interface changed from 'improved design' in 2000 and is currently 'optimized design'. Keywords such as evaluation system, evaluation index, hierarchical analysis, and mental model focus more on the computational aspect, indicating that research in recent years is gradually conducted by combining physiological indicators such as eye movement experiments with objective and subjective indicators such as user research.

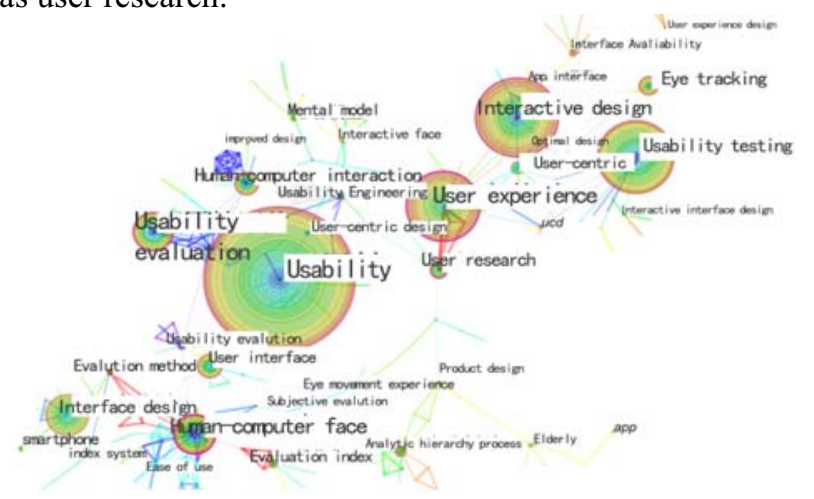

Figure 4: Domestic keywords

For example, Jiang Shan used hierarchical analysis to analyze the usability index values of the e-learning platform in her thesis, and conducted evaluation practice after establishing an evaluation system for the usability of the e-learning platform [4]. Mingzhuo Liu used usability measurement and evaluation to form a usability testing checklist for her own online course in Chapter 6 of her thesis [5]. Yuan Xiuli used the Delphi method, hierarchical analysis and neural network method, etc., and carried out some comparisons to make the setting of index weights more objective; the data of historical graduates were used to modify the system weights, and the relevant parameters and weights of the system were constantly modified and improved to make the evaluation system calculation results more close to the actual values [6]. 


\subsection{Analysis of foreign research hotspots}

A keyword co-current analysis was performed on 176 articles retrieved from the web of science, and according to the frequency of the keywords, the hotspots of foreign research on interface usability were obtained.
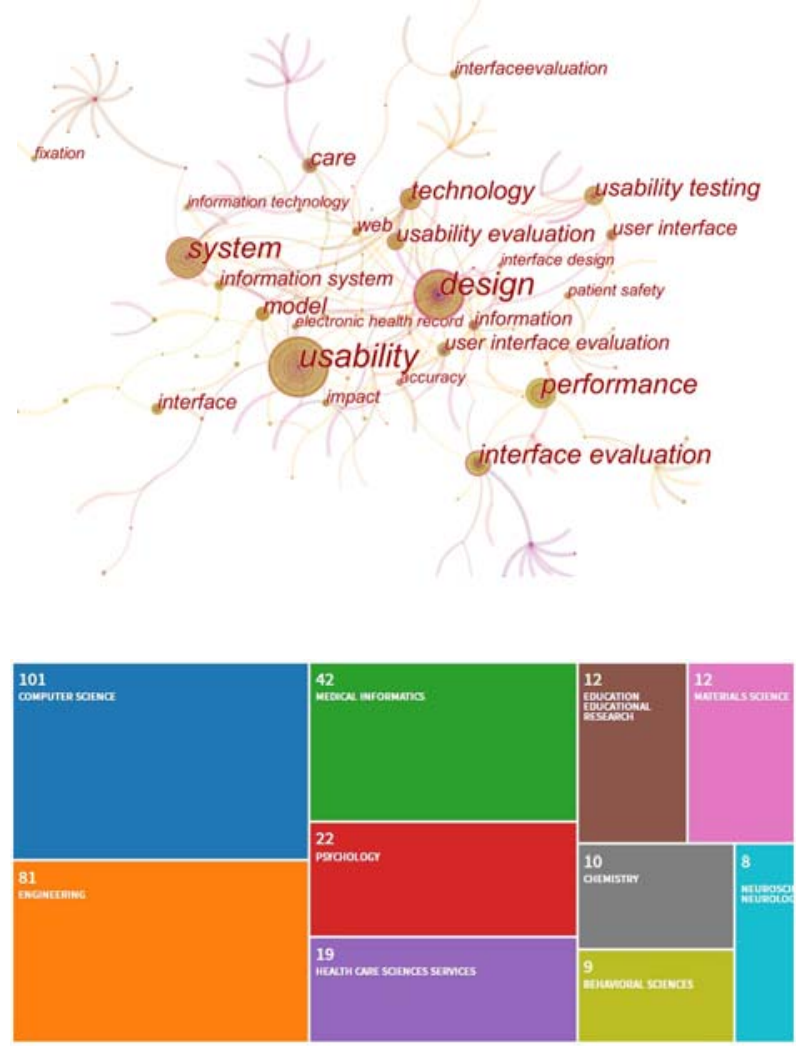

Figure 5: Foreign Keywords and Extended Analysis Chart

The bibliometric platform was used to cluster core terms, and the frequency table and core term clustering map were used to remove some of the repeated core terms, with usability evaluation, systems, healthcare, and children being high frequency terms. user-related and experimental terms became more frequent after 2013, such as user experience, user-centered design, usability testing, and empirical study of interaction design. Extended keywords have more related to algorithms: frameworks, models, computers, etc.

\subsection{Keyword emergence and keyword time zone graph analysis}

In this paper, CiteSpace's keyword emergence and keyword time zone map functions are used to discover the prominent keywords in recent years so as to draw relevant research trends. It is clear that the research on user experience in recent years is more extensive in China, and the keywords such as user research and mental model appear more frequently in 2018, which indicates that the research on interface usability in China is starting to favor the research on users based on the test.

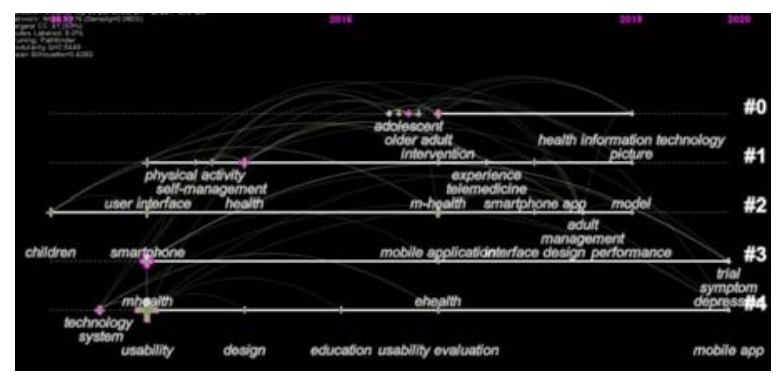

Figure 6: Domestic and international keyword time zone chart analysis

While the foreign research on interface usability content is divided into 2 major directions: medical direction and education direction, the medical aspect mainly focuses on the elderly, telemedicine, health information, self-management, etc., while the education aspect mainly focuses on children's education and app usability evaluation.

\section{Indicators and methods for assessing usability}

Usability is a cross-disciplinary theory and a topical issue that can be studied across disciplines, and the vagueness of the discipline makes it difficult to develop usability research. Usability can be used to describe users' performance, satisfaction, and ease of learning of a product, or all three of these dimensions at the same time, which makes accurate evaluation of usability difficult. [6]

Usability testing is a key element in the interface design process, helping designers to design and improve interfaces. Reasonable evaluation indexes and effective and reliable evaluation methods determine the later improvement of interfaces and even the improvement and optimization of system processes.

\subsection{Usability evaluation indexes}

The main evaluation metrics are efficiency, ease of learning, effectiveness, satisfaction, memorability, navigability, ease of use, error rate, aesthetics, and cognitive load; the most commonly used are efficiency, effectiveness, and satisfaction, and the post-optimized and pre-optimized interfaces are generally compared through these three metrics.

The research on user experience has increased, and some of the similar indicators have been conceptually overlapped, with some papers suggesting that ease of learning includes low cognitive load, ease of use includes easy to remember, and efficiency and satisfaction are divided into more indicators. For example, efficiency was expanded to include search speed and minimum action, and satisfaction was expanded to include aesthetics, cognitive load, simplicity, and error handling. The main problems that prevent the user experience evaluation index system from being standardized and unified are inconsistent classification standards, inconsistent naming of indexes, and cross-concept. 


\subsection{Interface usability evaluation methods}

Interface usability evaluation methods include subjective evaluation method, performance evaluation method, mathematical model evaluation method, comprehensive theory evaluation method and physiological experiment evaluation method. The first four methods are traditional evaluation methods, which are more mature and perfect in practical application, but are greatly influenced by subjective factors. In contrast, the physiological experimental assessment method is more scientific, objective and stable, and can provide quantitative responses to the user's real-time physiological data. Through eye-tracking and electroencephalography, the visual cognitive strategies and neurophysiological indicators of the subject's interface information can be obtained and mutually verified from the exogenous physical stimuli to the endogenous physical responses, revealing the user's cognitive pattern of the digital tanning interface. [7] Therefore, by integrating various evaluation methods, we can make up for the shortcomings of each method and identify the deficiencies in the interface, so as to guide and improve the design of the interface.

In the research on objective evaluation methods of interface usability, eye-tracking technology and EEG technology have become the hotspot and focus of domestic and international research.

\section{Concluding remarks}

Through the analysis of domestic and foreign papers on interface usability, combined with CNKI and WOS bibliometric analysis tools and CiteSpace information visualization software to analyze the number of publications, literature sources, institutions, authors, keywords and other aspects, the current research status, trends and corresponding indicators and methods of interface usability were found, with emphasis on the evaluation indexes and methods of interface usability. The study shows that user research and usability testing are important decision factors for interface design. The existing studies have some differences in the evaluation indexes and methods for interface usability, and most of them are directly chosen by expert methods. Objective measurement and quantitative evaluation methods of interface usability still need to be improved.

At present, there are still the following deficiencies in the research on user experience: the application field of usability should be expanded continuously, not only to the interface, but also to the usability of the whole app or system process. The description and study of long-term user experience and interaction environment are relatively simple, and an effective evaluation system of interaction environment is lacking. The purpose of interface usability research is to find out the design elements that affect interface usability, and to improve the level of interface usability by optimizing the design, which will ultimately increase user satisfaction. [8]

Therefore, the following points should be paid attention to in future research: continuously expand the application field of interface usability research, start with usability experiments, and study the components, measurement methods and evaluation models of interface usability according to the characteristics of users, especially the evaluation methods that integrate various evaluation indexes; considering the dynamic nature of interface usability, the definition, composition, measurement and evaluation methods of long-term experience will be a focus of future research. Interface usability research involves knowledge from physiological, psychological, behavioral sciences, perceptual engineering, and decision sciences; therefore, the psychological and physiological perceptions of users should be studied in conjunction with the research results from these disciplines in interface usability measurement and evaluation research.

\section{REFERENCES}

1. Pan Fei, Jiang Ke, Wang Dongqi. Research on usability design of ticketing website based on eyetracking technology[J/OL]. Packaging Engineering:18[2020-05-26].

http://kns.cnki.net/kcms/detail/50.1094.TB.20200309 .0941.002.html.

2. He Jiajie. An overview of usability testing user affective experience evaluation scale[J]. Journal of Consumerism, 2018(11).

3. Wu, Changxu, Zhang, Kan. Diagnostic User Interface Usability Evaluation Method (IM) (Upper)Introduction and Evaluation[J]. Ergonomics,2000(03):54-57.

4. Jiang Shan. Research and application of e-learning platform usability evaluation system [D]. Beijing University of Posts and Telecommunications, 2014.

5. Liu Mingzhuo. Research on the Usability of Elearning Courses [D]. East China Normal University, 2010.

6. Yuan Xiuli. Research and Practice on the Evaluation System of Computer Science Students' Competency Achievement Based on Engineering Education Certification Standards[D]. Shenyang Normal University,2019.

7. The golden 8 rules of classic user interface interaction design [EB/OL]. [2013-08-31]. http://www.360doc.com/content /10/0121/17/748592_14102159.shtml.com/content/1 0/0121/17/748592_14102159.shtml.com/content/10/ 0121/17/748592_14102159.shtml.

8. Niu Yafeng. Research on the evaluation method of digital interface usability based on EEG technology [D]. Southeast University, 2015. 